\title{
The Daphnia bioassay: a critique
}

Donald J. Baird, Ian Barber, Mairead Bradley, Peter Calow \& Amadeu M.V.M. Soares

Department of Animal and Plant Sciences, University of Sheffield, Sheffield S10 2TN, U.K.

Key words: Daphnia, bioassay, effects of food concentration, genetic variation, maternal effects, laboratory culture, genotype-environment effects

\begin{abstract}
Daphnia magna is used widely as a standard ecotoxicological indicator organism, and protocols exist for its use in assessing the toxicity of substances under acute and chronic experimental conditions. Problems exist in repeatability of such bioassays between laboratories. Sources of variation are identified using a simple quantitative genetics model. Presenting specific examples, we conclude that these problems are tractable, but only if the genotype and culture conditions prior to and during tests are strictly controlled.
\end{abstract}

\section{Introduction}

One of the essential properties of a laboratory bioassay is that it should be repeatable. This is true whether single-species or multi-species bioassays are being considered. In this paper we specifically evaluate problems which cause inconsistencies among single-species bioassays that use the freshwater cladoceran Daphnia magna Straus, but emphasise that the conclusions drawn are applicable to all types of ecotoxicological tests, regardless of species or compound being considered.

D. magna is used in aquatic toxicology primarily for its ease of culture, its high sensitivity to toxicants and its clonal method of reproduction (Berge, 1978; Adema, 1978). Two types of bioassays are commonly performed with it: 48 -hour acute tests using neonates that are $<24$ hours old, and 21-day chronic life-cycle tests that are run from birth to ca. the tenth instar. In the former, the toxicological effect is death; in the latter it is the inhibition of 'normal reproduction'.

\section{The problem}

Within the European Community (EC), bioassays are periodically checked by the 'ring test' procedure in which different laboratories follow similar protocols to assess reference toxins. The results provide a measure of interlaboratory consistency in the performance of a standard bioassay.

Such a ring-test, involving a 21-day life-cycle test with sodium bromide as reference toxin, has recently been carried out using D. magna (Cabridenc, 1986). The criterion used was the noeffect concentration, defined as the concentration immediately below that in which a significant effect on reproduction occurred. Of 37 participating laboratories, 22 returned results which satisfied validity criteria specified in the protocol. Most tests which failed validity criteria did so because of low fecundity in the control treatments (see also below). The final results (Table 1) exhibit the maximum possible variability in response. We believe that this extreme inconsistency illustrates a serious problem that can only be solved by a 
Table 1. Results of the $1986 \mathrm{EC}$ ring test on the chronic effects of sodium bromide on D. magna fecundity (After Cabridenc, 1986).

$\begin{array}{ll}\text { Laboratories participating: } & n=37 \\ \text { Valid test results obtained: } & n=22 \\ \text { NaBr concentration range: } & 3-117 \mathrm{mg} \cdot \mathrm{L}^{-1} \\ \text { Range of results obtained: } & <3->117 \mathrm{mg} \cdot \mathrm{L}^{-1} \\ \text { (NOEC on fecundity - see text) } & \end{array}$

systematic examination of each of the variability components of the toxicological response.

\section{The model}

We adopt a standard quantitative genetics approach to this problem (Falconer, 1981), viz.:

$$
V_{P}=V_{G}+V_{E}+V_{G E}
$$

This equation partitions the total variability in stress-response of a group of test animals $\left(V_{P}\right)$ into three subcomponents:

(1) $V_{G}$, variability due to genetic heterogeneity

(2) $V_{E}$, the variability due to environmental heterogeneity.

(3) $V_{G E}$, the variability due to genotype by environment interaction.

Using this approach, we not only make suggestions for improvement of culture systems and future test protocols, but also comment on the suitability of clonal organisms for use in ecotoxicology.

\section{Genetic variability}

Genetic typing of the clones used by the EC ringtest laboratories has been carried out (Bradley et al., in prep.) using standard electrophoretic techniques (Hebert \& Beaton, 1986). The details will be published elsewhere, but a summary is given briefly in Fig. 1. Genetic differences between laboratory stock clones appear to have arisen for two reasons. Firstly, a number of labo-

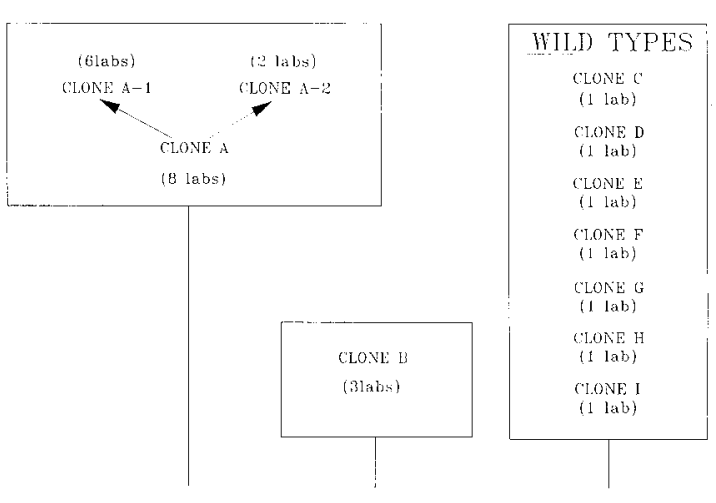

FUROPEAN TESTING LAI3 CLONFS

Fig. 1. Results of the EC interlaboratory genetic typing exercise. 27 laboratory clones typed at six enzyme loci (PGM, PGI, MPI, GOT, MDH-2, ES-1) from a sample of 30-40 animals drawn randomly from laboratory stock cultures.

ratories obtained stocks directly from local wild populations (clones $\mathrm{C}-\mathrm{I}$ ), and, not surprisingly, these have proved genetically unique. Secondly, some genetic heterogeneity has been due to clonal divergence (clones A-1 and A-2 being direct descendants of clone A). Here, a number of laboratories received their stocks from a central source that at some point gave rise to two subclones, either due to mutation or sexual recombination. The former seems rather more likely than the latter as the source of clonal divergence, since the typing study failed to reveal any within-laboratory genetic variation in stocks - each consisted of a pure clone.

In a standardised series of acute toxicity tests (48 $\mathrm{hr} \mathrm{LC}_{50}$ tests following a standard protocol (OECD , 1981)) using cadmium chloride (concentrations measured using graphite furnace atomic absorption spectrophotometry), we have found (Baird et al., in prep.) the range in response of the ring-test laboratory clones ranged from $0.8 \pm 1.1 \mathrm{ppb}$ to $25.8 \pm 1.1 \mathrm{ppb}$ (mean \pm s.e.). This clearly indicates that differences in genotype do reflect significant differences in toxicological response. Hence the results obtained from $D$. magna bioassays may depend on the clone chosen to run the test. 


\section{Environmental variability}

We can recognise two separate components of environmental variability: the conditions experienced prenatally in the mother ('maternal effects', Falconer, 1981), and conditions experienced by individuals after birth. In practice these two components refer respectively to conditions in stock culture and during the bioassay itself.

Since there is as yet no standardisation between different laboratories in culture methodology, it is likely that maternal effects could be an important source of variability in bioassay results. It is well-known that maternal reserves play an important role in juvenile growth for Daphnia (Tessier et al., 1983), but of greater importance is whether maternal provisioning influences the toxicological response of offspring. To examine this, mothers from a single clone were raised under constant culture conditions (following guidelines in Goulden et al., 1982), but at two different ration levels $\left(0.05\right.$ and $0.5 \mathrm{mgC} \mathrm{L}^{-1}$ Chlorella vulgaris in axenic culture), and their offspring $(<24 \mathrm{hr}$ old) were compared in a standard $48 \mathrm{hr} \quad \mathrm{LC}_{50}$ test using 3,4-dichloroaniline. The results (Fig. 2) indicate that offspring from high-ration mothers were approximately twice as susceptible to 3,4-dichloroaniline as those from low-ration mothers $\left(\mathrm{LC}_{50}\right.$ : $104 \pm 4.2 \mathrm{ppb}\left(0.5 \mathrm{mg} \mathrm{C} \cdot \mathrm{L}^{-1}\right)$ versus $195 \pm 6.3$

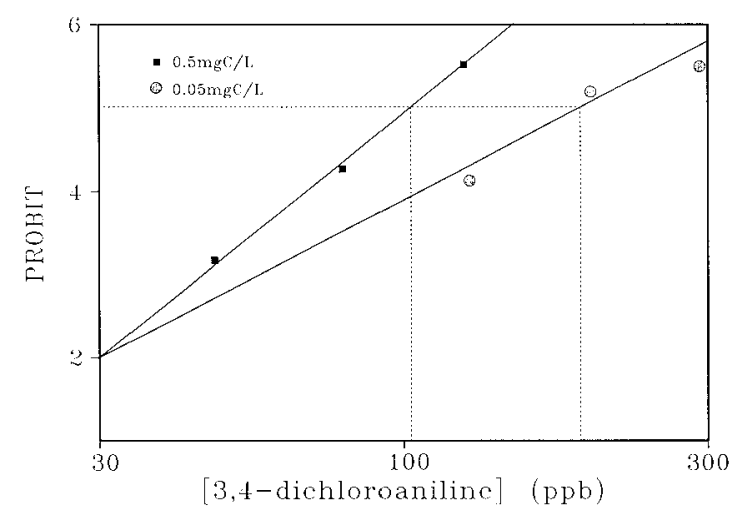

Fig. 2. The influence of maternal ration level on $48 \mathrm{hr}$ acut $\epsilon$ response (measured as $\mathrm{LC}_{50}$ - dashed lines) of $<24 \mathrm{hr}$ old neonates to 3,4-dichloroaniline. $\left(0.05 \mathrm{mg} \mathrm{C} \cdot \mathrm{L}^{-1}\right)$. Maternal effects are therefore of toxicological importance, particularly in neonate $48 \mathrm{hr}$ acute tests, and worthy of more detailed investigation.

The second component of environmental variability relates to conditions in the bioassay itself. The EC ring-test provides a good example of this. From these data, it is clear that as ration level increases, fecundity increases (Fig. 3). In the EC ring-test, one of the validity criteria specified was a minimum fecundity of 70 neonates per animal in the control treatment. Many laboratories failed this criterion. From the same relationship of fecundity and ration level for the control animals in the EC ring-test (Fig. 4), two conclusions emerge: firstly, the specified ration level of $1.0 \mathrm{mg}$ $\mathrm{C} \cdot \mathrm{L}^{-1}$ was not always given (actual range $0.1-100 \mathrm{mg} \mathrm{C} \cdot \mathrm{L}^{-1}$ ), and secondly, that in those tests that were run at the correct ration level, fecundity ranged from 9 to 129 neonates per adult female. Of the variability in fecundity, almost $64 \%$ can be explained by a failure to adhere to the specified ration level. Hence it is not surprising that the ring-test results showed so much variability.

\section{Genotype-environment interactions}

Given that clones of D. magna exhibit different tolerances to a specified toxicant (see above), it

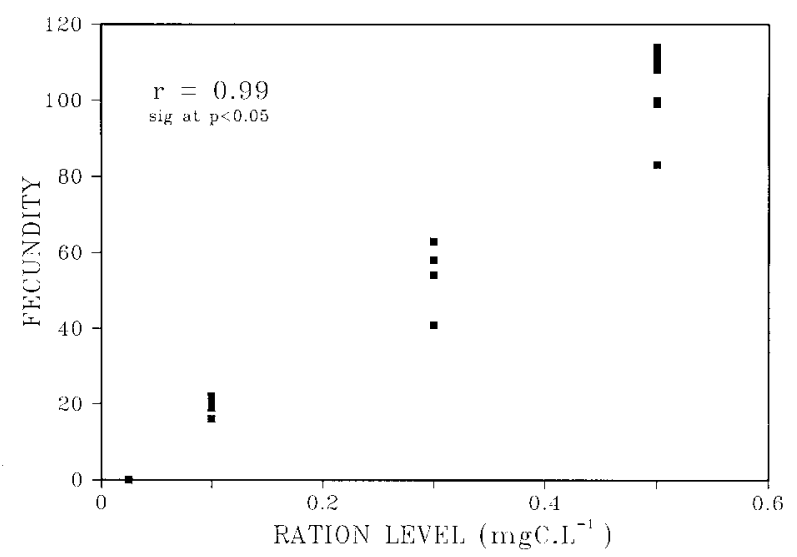

Fig. 3. Fecundity of D. magna over 21 days in unstressed conditions at different ration levels. (Food $=$ Chlorella vulgaris maintained in axenic culture). 


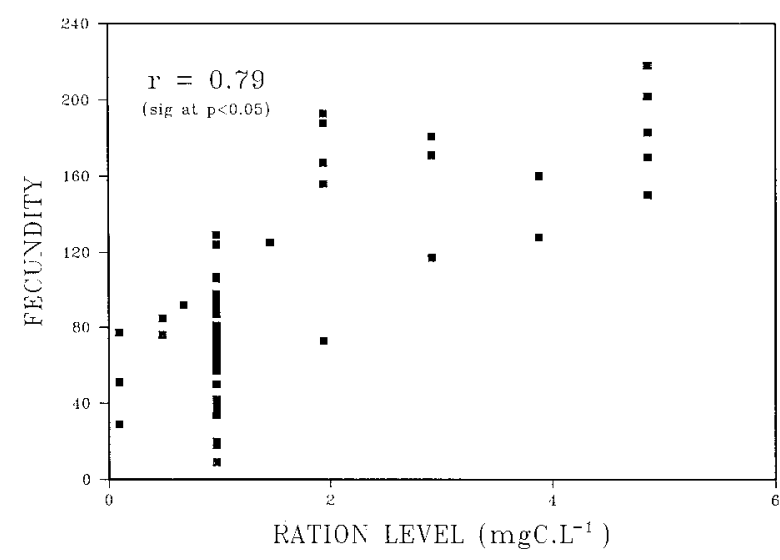

Fig. 4. Fecundity of D. magna in control (i.e. unstressed) treatments in the $1986 \mathrm{EC}$ ring test as a function of ration. N.B. One point (ration $=100 \mathrm{mgC} \cdot \mathrm{L}^{-1}$, fecundity $=0$ ) has been omitted to improve clarity.

would be useful to know if the rank order of tolerance among clones reared under similar conditions is the same for different classes of toxins, and to what extent interclonal differences in stress-tolerance reflect differing degrees of adaptation to the laboratory environment. These questions address the problem of subtle interactive effects between genotype and environment about which we know little but which certainly merit further investigation.

It follows that there are two ways in which two laboratories may obtain differing results in a standard test even when adopting constant culture conditions: by culturing different genotypes under the same constant environmental conditions, or by culturing the same genotype under different environmental conditions, irrespective of the constancy of those environments. Since neither genotype nor culture environment are at present specified for laboratories running standard D. magna bioassays, between-laboratory variability is not surprising.

\section{Summary}

i) To improve consistency in the D. magna bioassay among testing labs, both genotype and culture conditions must be specified. Test protocols must be unambiguous; if tests are carried out under conditions that deviate from the protocol the results must be discarded.

ii) Further research into each of the components of equation (1) should identify which clone/s is suitable for testing purposes, and should lead to the formulation of 'good laboratory practice' guidelines.

iii) While in principle it should be straightforward to minimise $V_{G}$ and $V_{E}$, the interactive effects arising from $V_{G E}$ are likely to be subtle and merit more detailed investigation.

\section{Acknowledgements}

Portions of this work were funded by NERC grant TSF $/ 86 / \mathrm{AeT} / 4, \quad \mathrm{EC}$ contracts $\mathrm{CCAM} / 87 / 319$ and $\mathrm{B} / 86000160$ and a NATO grant $3 / \mathrm{A} / 87 / \mathrm{PO}$.

\section{References}

Adema, D. M. M., 1978. Daphnia magna as a test animal in acute and chronic toxicity tests. Hydrobiol. 59: 125-134.

Berge, W. F. ten, 1978. Breeding Daphnia magna. Hydrobiol. 59: 121-123.

Cabridenc, R., 1986. Exercice d'intercalibration concernant une méthode de détermination de l'ecotoxicité à moyen terme des substances chimiques vis-à-vis des daphnies. Unpublished EC Report. Contract W/63/476 (214). Ref. I.R.C.H.A.D. 8523 . Vert-le-Petit, France. 20 pp.

Falconer, D. S., 1981. Introduction to quantitative genetics. Longman. Harlow, Essex. 340 pp.

Goulden, C. E., R. M. Comotto, J. A. Hendrickson Jr., L. I Horning \& K. L. Johnson, 1982. Procedures and recommendations for the culture and use of Daphnia in bioassay studies. Pages 139-160 in Proceedings of the American Society for Testing and Materials Fifth Toxicology Symposium, Special Technical Publication Number 7668, Philadelphia, Pennsylvania, USA.

Hebert, P. D. N. \& M. Beaton, 1986. Cellulose-acetate gel electrophoresis. Unpublished ms. Univ. Windsor, Ontario. $34 \mathrm{pp}$.

OECD., 1981. Daphnia sp. 14 day reproduction test (including acute immobilisation test). OECD guidelines for testing of Chemicals, no. 202. ISBN 92-64-1221-4. Paris. 15 pp.

Tessier, A. J., L. L. Henry, C. E. Goulden \& M. W. Durand, 1983. Starvation in Daphnia: Energy reserves and reproductive allocation. Limnol. Oceanogr. 28: 667-676. 